\title{
EL PAPEL DEL ISLAM EN EL PENSAMIENTO FEMINISTA DE ṬĀHAR AL-ḤADDĀD
}

\section{THE ROLE OF ISLAM IN ȚĀHAR AL-HAADDĀD'S FEMINIST THOUGHT}

\author{
Tatiana HERNÁNDEZ JUSTO \\ Universidad de Granada
}

Recibido: 15/7/2016

Aceptado: 05/09/2016

Para citar este artículo / To cite this article:

Hernández Justo, Tatiana. «El papel del Islam en el pensamiento feminista de ṬĀHAR AL-HADDĀD». En Nieves Montesinos Sánchez y Beatriz Souto Galván (coords.), Laicidad y creencias. Feminismo/s, 28 (diciembre 2016): 133-146, DOI: $10.14198 / \mathrm{fem} .2016 .28 .05$

Para enlazar con este artículo / To link to this article:

http://dx.doi.org/10.14198/fem.2016.28.05

\section{Resumen}

Ṭāhar al-Haddād (1899-1935), adalid del feminismo en Túnez, dedicó su más importante obra a describir y criticar la situación de las mujeres en su país en busca de mejoras que acabaran con la desigualdad entre hombres y mujeres. Esta obra, Imra'tu-nā fi l-Šarĩca wa-l-muŷtamac, le costó la expulsión de la academia además de serios problemas con la institución religiosa más influyente de su país, la universidad islámica al-Zaytuna, a pesar de que sus teorías se enmarcaban claramente en un ámbito religioso. A lo largo de este trabajo estudiaremos el papel de la fe islámica en el pensamiento de Ṭāhar al-Ḥaddād a través del análisis de las propuestas ofrecidas por el autor en su obra, determinando rupturas y continuidades con la tradición islámica del momento.

Palabras clave: Túnez, Ṭāhar al-Ḥaddād, islam, feminismo, reformismo. 


\begin{abstract}
Ṭāhar al-Ḥaddād (1899-1935), beginner of feminism in Tunisia, was a towering figure who dedicated his best known work to describing and criticizing the edging situation women in his country lived in, while offering his own views on what had to be done to stop gender inequality. Said work, Imra'tu-nā fi l-Šarīca wa-l-muŷtama ${ }^{c}$, became a major discussion topic in the society of his time and cost him several problems with al-Zaytuna, Tunisia's biggest religious institution, even though al-Haddad's theories come from a clearly religious background. Throughout this article we'll study the role of Islamic faith in his thought via the analysis of the proposals offered in his book, searching for continuity and rupture with the Islamic tradition of his time.
\end{abstract}

Keywords: Tunisia, Țāhar al-Ḥaddād, Islam, feminism, reformism. 


\section{INTRODUCCIÓN}

La figura de Ṭāhar al-Ḥaddād (1899-1935), pensador y reformista tunecino clave para comprender el proceso de emancipación y empoderamiento femenino en el país, ha sido objeto de pocos estudios, tanto dentro como fuera de las fronteras nacionales. Este autor, conocido principalmente por su faceta como luchador a favor de las mujeres pero también muy implicado con la causa nacionalista y el movimiento obrero, fue duramente criticado en su época, especialmente por sectores conservadores dentro del ámbito religioso, e incluso llegó a ser acusado de herejía. Su obra más importante en la materia, titulada Imra'tu-nā fi l-Šañ̄a wa-l-muŷtamac (Nuestra mujer en la ley islámica y la sociedad), recibió tales críticas que se prohibió inmediatamente su circulación y el autor fue obligado a abandonar la Escuela Superior de Derecho, donde se encontraba estudiando, y a entregar su título de al-Zaytūna (Sraïeb 106-107).

Sin embargo, un primer vistazo a la obra nos permite comprender el cariz reformista de la misma sin llegar a percibir un carácter rupturista con el islam. Al hacerlo, nos percatamos de que este libro se enmarca en la corriente comenzada por eruditos como Ŷamāl al-Dīn al-Afgānī (1838-1897) y Muḥammad 'Abduh (1849-1905), una corriente reformista que promulga una nueva lectura del islam, adecuándola al contexto contemporáneo y a las nuevas circunstancias sociales e históricas, en lugar de un rechazo a la religión. ¿Cuál es el papel de la religión en el discurso de al-Ḥaddād? ¿Cuándo se da una ruptura con la tradición social y qué podría considerarse realmente como una crítica a la fe islámica? Para tratar de responder a estas preguntas, nuestro objetivo en este artículo es estudiar las propuestas ofrecidas en Imrātu-nā fi l-Šarĩ $a$ wa-lmuŷtama $a^{c}$ determinar cuál es el rol del islam en el pensamiento de Tāarar al-Haddād. Para ello, emplearemos tanto la versión original en árabe (Țāhar al-Ḥaddād 1989) como la traducción al inglés de la obra (Ronak Husni y Daniel L. Newman) y haremos especial hincapié en aquellas cuestiones que suscitaron mayor polémica en la obra y que más claramente podemos relacionar con el aspecto religioso del islam. 


\section{ANÁLISIS DE LA OBRA}

Al-Ḥaddād perfila a lo largo de toda su obra una serie de cuestiones transversales en torno a las cuales gira su pensamiento y que se encuentran presentes no sólo en Imrā'tu-nā fi l-Šarī'a wa-l-muŷtama ${ }^{c}$, sino en todos sus libros. Estos axiomas de su pensamiento son, por un lado, la importancia de la educación para la consecución de mejorías; por otro lado, la necesidad de una liberación nacional (como dijimos anteriormente, al-Haddād era un autor sumamente comprometido con la causa nacionalista) para que se produzcan las liberaciones sociales que él promulga (a saber, la liberación femenina y la liberación obrera) y, por último, la tesis de que el islam no es el culpable del atraso y el estancamiento que sufría su país en su época, sino que los hombres, a través de interpretaciones incorrectas y tradicionalistas, han desviado el verdadero significado de la Palabra de Dios. Este último punto es el que más nos interesa para analizar el rol de la religión en el pensamiento del autor, puesto que nos permite ver, en primer lugar, la importancia que la fe tiene para al-Ḥaddād así como su visión reformista al respecto, compartida por otros autores coetáneos, como el conocido Qāsim Amīn (1863-1908).

Para al-Ḥaddād, esta situación de atraso en que se ha sumido la sociedad tunecina (y la sociedad islámica en general) se ha debido a la intrusión en la religión de prácticas sociales o culturales que son ajenas al mensaje divino y que han persistido a causa del machismo de sus intérpretes y de la ignorancia de los creyentes. Por ende, es necesario purificar el islam y extraer de él todos estos elementos exógenos, además de adaptar el texto a su contexto para que éste se pueda reinterpretar y pueda ser comprendido y vivido por la sociedad del momento. Para ello, el autor se muestra partidario de hacer una doble distinción en cuanto a las normas comprendidas dentro de la ley islámica: por un lado, aquellas leyes que son mutables y permiten la reinterpretación y la introducción de reformas y, por otro lado, las normas que serían inalterables y no admitirían ningún tipo de modificación. Para él, las leyes referidas a la situación de las mujeres pertenecen al primer apartado y, por tanto, es lícito revisar la legislación vigente para perfeccionarla y permitir que las mujeres recuperen el puesto que, según al-Ḥaddād, el Corán y Dios les confirieron. Partimos, por tanto, de un pensamiento de raíz religiosa que no combate la fe, sino que le confiere el privilegio de haber sentado las bases de la igualdad, aunque luego la práctica religiosa y los creyentes desfiguraran esta cuestión. Por ello, las críticas de al-Ḥaddād siempre van a enmarcarse en lo que el autor considera como el «correcto camino» dentro de la interpretación islámica y la religión va a ser la que marque las pautas de lo que es lícito e ilícito en materia de emancipación y participación femenina. 
Al-Haddād critica duramente costumbres culturales como el velo (especialmente el velo facial, que era un símbolo del enclaustramiento), la reclusión de las mujeres en las casas, la falta de contacto entre los sexos, la ausencia de mujeres en la vida pública, los matrimonios concertados, etc. Al mismo tiempo, pone de manifiesto las diferencias de opinión entre orientales y occidentales acerca del lugar de la mujer en la sociedad. Mientras en Occidente las mujeres son educadas y trabajan codo con codo con los hombres en busca del progreso de sus naciones, el autor argumenta que en Oriente las mujeres se hallan relegadas a una posición inferior, siempre dentro de los límites del hogar y sin posibilidades reales de formar parte activa de la sociedad y del progreso de sus países. Las causas de esta situación son completamente ajenas a la esencia misma del islam que, en realidad, supuso un gran avance para la situación de las mujeres en la época preislámica al concederles el derecho, entre otras cosas, a tener una dote y a participar de la herencia que dejaran sus parientes. De entre todas esas causas, al-Haddād hace especial hincapié en una, la más importante a su juicio, que es la interpretación que los hombres hacen de la religión y, más concretamente, del Libro Sagrado.

\section{APARTADO JUDICIAL}

A lo largo de las dos grandes partes en que se halla dividida la obra (un apartado para las cuestiones jurídicas y otro para las sociales), al-Ḥaddād repasa aquellas cuestiones que considera necesitadas de reforma y comienza hablando del testimonio y la judicatura. Para el autor, el hecho de que el Corán no mencione explícitamente la posibilidad de que las mujeres ocupen cargos judiciales no es sinónimo de que esté prohibido, sino que se debe a la falta de preparación que tenían las mujeres de la época y que les impedía desempeñar dichas funciones. Por lo tanto, cualquier mujer que sea instruida para el cargo puede ocuparlo sin perjuicio del Corán y la ley de Dios, ya que el Libro Sagrado dice explícitamente que los hombres y las mujeres tienen las mismas capacidades. Además añade que, en la sociedad tunecina de su época, las mujeres no tenían derecho a ocupar una dignidad judicial y no estaban acostumbradas a estar junto a un hombre testificando ante un juez, lo cual justifica que el Corán estableciera que el testimonio de una mujer tuviera la mitad de valor que el de un hombre. Por este motivo, lo único que se necesita para que las mujeres puedan tener acceso a este tipo de cargos es que se las instruya y prepare para ello.

El Corán da a las mujeres los mismos derechos civiles que a los hombres, tales como el derecho a la propiedad o a la herencia, como puede leerse en múltiples aleyas citadas por al-Ḥaddād, por lo que resulta una contradicción obligarlas a permanecer en sus casas y no permitirles disponer de sus 
propiedades como deseen. Esta costumbre del enclaustramiento, según el autor, tiene sus orígenes en la sociedad y en la cultura y no en la ley islámica y, por tanto, debe ser abolida. Al-Haddād critica la falta de libertad que sufren las mujeres y la imposición arbitraria del velo, argumentando que esto no puede deducirse del Libro Sagrado, sino que se trata de una mala interpretación que ha perpetuado las tradiciones previas al advenimiento de la religión. Una vez más, la fe islámica se muestra como adalid de los derechos de la mujer frente al periodo anterior a la Revelación.

Es cierto que el Corán establece unas leyes de herencia determinadas que dejan a la mujer la mitad que a cualquier varón en su mismo grado de parentesco (Corán, IV:11), pero el hecho de que existiera una aleya que obligue a dejar una parte de la herencia para las mujeres era ya de por sí algo positivo, teniendo en cuenta que antes de la llegada del islam las mujeres no sólo carecían de este derecho sino que formaban parte de dicha herencia. Sin embargo, el autor critica que esta ley siga aplicándose tal y como figura en el Libro Sagrado ya que este aspecto de la religión pertenecería al grupo de leyes que pueden ser modificadas, como ocurrió en su momento con la esclavitud.

Ṭāhar al-Ḥaddād defiende la necesidad de combatir la fornicación, prohibida por el islam, con la instrucción y la educación de la mujer más que con castigos físicos. Para él, la verdadera manera de atajar este pecado no es reprendiéndolo, sino promoviendo el matrimonio, creando una vía que conecte a hombres y mujeres y educando a los futuros cónyuges en la moral de la nación y en los deberes matrimoniales. El autor define el matrimonio como un vínculo que debe establecerse tomando como base el amor, el respeto mutuo, el deseo de procrear y el conocimiento de los deberes conyugales. En este sentido, defiende y avala con citas coránicas que la mujer debe dar su consentimiento libre y voluntario acerca de cualquier tema de esta índole, denunciando las situaciones en que los padres y tutores legales de jóvenes impúberes escogen un esposo basándose en los beneficios que obtendrán del enlace y no en lo mejor para las niñas.

También critica el concepto de unión carnal como placer exclusivo del hombre, es decir, la idea de que el varón es el único que debe disfrutar con la unión sexual, o que las mujeres no puedan divorciarse de sus maridos si son impotentes, cosa que sí podía hacerse a la inversa. Para él, es evidente la manifiesta injusticia de esta ley, que convierte a las mujeres en objetos sexuales para disfrute de los maridos y les impide a obtener un placer que el Corán les otorgó.

En cuanto al impedimento de que las mujeres se casen con hombres no musulmanes, al-Ḥaddād sostiene que la causa de esta ley no es una injusticia contra las mujeres sino que se trata de una forma de salvaguardar el número 
de musulmanes, ya que la religión se transmite por vía paterna y se considera musulmán a cualquier niño de padre creyente. El objeto de la ley sería aumentar el número de musulmanes al mismo tiempo que evitar el crecimiento de creyentes de otras religiones. Sin embargo, el autor afirma que los hombres que contraen matrimonio con mujeres de otras religiones y nacionalidades producen el mismo efecto, pues si bien sus hijos son considerados musulmanes muchos no lo son en realidad, sino que toman de sus madres sus costumbres y religiones y de esta forma, la nación se debilita junto a la religión. En este sentido, para entender mejor su pensamiento, hay que tener en cuenta que la sociedad tunecina estaba sometida a una potencia extranjera y la homogeneidad de las familias era percibida como un signo de fortaleza y de unidad frente al invasor francés.

Siendo uno de los puntos clave de la lucha de Țāhar al-Ḥaddād y uno de los que encontraron respuesta cuando se alcanzó la independencia y se promulgó el Estatuto Personal de Túnez, puesto que fue abolida de inmediato, a la poligamia dedica al-Ḥaddād un extenso apartado en su obra. En él demuestra que el Corán la prohíbe puesto que la condición para que ésta sea lícita es imposible de cumplir. La tradición había ignorado las partes del Corán donde se aclaraba bajo qué circunstancias era lícita y bajo cuáles no y había transformado la institución en un derecho de los hombres siempre y cuando fueran equitativos con sus esposas en el aspecto material. No obstante, el Libro Sagrado estipula que el único caso en que se puede ejercer la poligamia es cuando el marido sea justo con todas sus esposas y acto seguido afirma que es imposible que esta condición se cumpla ${ }^{1}$.

Al-Haddād trata el tema del repudio y critica el abuso que se hacía en su época del triple repudio (fórmula por la cual una mujer y su marido pasaban a estar totalmente divorciados y no podían volver a contraer matrimonio si la mujer no se casaba con otro hombre antes). En su época era muy frecuente la contratación de un hombre como segundo marido por parte del primero, de tal forma que este «falso marido» recibía un sueldo a cambio de casarse con la esposa y volverla a repudiar para que ésta estuviera nuevamente disponible para el primer esposo. Según el autor, esto constituye un insulto al sentido del repudio y a la mujer en sí, de modo que es necesario combatir estos abusos

1. Las aleyas a las que nos referimos son IV:3 y IV:129. En la primera se presenta la poligamia como una forma de ser justo con los huérfanos y las viudas y se aclara que esta práctica sólo puede llevarse a cabo cuando el marido sea justo con todas las mujeres por igual, pero en IV:129 se dice que los hombres no podrán ser justos aunque quieran, de modo que es imposible que se cumpla la condición de justicia necesaria para llevar a la práctica la poligamia. 
erradicando el poder de los maridos de repudiar a sus mujeres a voluntad. Para ello propone la institucionalización del divorcio legal como única vía de poner fin a un matrimonio, es decir, el paso obligatorio por el tribunal para que el proceso sea oficial y efectivo. De esta manera, los hombres dejarían de estar en posesión de ese derecho exclusivo y no abusarían del mismo al tiempo que las mujeres obtendrían el derecho a pedir el divorcio.

También se denuncia la injusticia que se cometía sistemáticamente en Túnez contra las mujeres repudiadas, a las que se negaba el derecho a recibir una indemnización pecuniaria cuando eran expulsadas por sus maridos, incluso cuando esto aparecía en el propio Corán. Para al-Haddād esta cuestión responde a una interpretación sexista y arbitraria del Corán, hecha para favorecer a los hombres, puesto que se entendía que la única indemnización obligatoria era la de dar alojamiento y alimento a la mujer repudiada durante el periodo de espera legal. Para combatir dicha injusticia promueve la necesidad de observar de manera más estricta estas aleyas coránicas y de aplicarlas con equidad en todas las situaciones puesto que, en sus propias palabras, «si volvemos a las bases islámicas sobre multas por perjuicios veremos que de ellas sale este reconocimiento (de dar a la mujer una indemnización por el repudio)» (Ṭāhar al-Ḥaddād 77).

Para respaldar lo expuesto en esta parte el libro, el autor incluye una serie de preguntas y respuestas hechas a grandes maestros de jurisprudencia islámica, tanto maliquíes como hanafíes (las escuelas coránicas con mayor presencia en Túnez en aquella época), a fin de que dieran su opinión sobre algunos de los temas tratados en la obra, como el derecho de la mujer a escoger su esposo o el derecho al divorcio por petición de la esposa. El objetivo de este apartado de preguntas y respuestas era demostrar que sus opiniones no eran las únicas a favor de la liberación de las mujeres, sino que muchos otros estudiosos estaban de acuerdo en conferirles una mayor libertad y en excluir del mensaje divino prejuicios históricos. Esto y las numerosas citas coránicas presentes en todo su libro sirvieron a sus defensores para justificar que en ningún caso pretendía criticar el islam ni el Corán, sino las interpretaciones erróneas y anquilosadas que se habían ido perpetuando siglo tras siglo, pero dichas justificaciones no fueron suficientes para detener la miríada de críticas que llovieron sobre el libro y sobre el propio autor (por ejemplo, poco tiempo después de que se supiera que la obra iba a ser publicada comenzaron a aparecer libros dedicados a refutar las tesis del autor, como recoge Borrmans (138-146)). 


\section{APARTADO SOCIOLÓGICO}

En esta segunda parte se tratan todos los temas relativos a la sociedad tunecina que afectan a la mujer y las causas y consecuencias de muchos de los males sociales que aquejan a las mujeres y les impiden progresar.

En la época en que se compuso esta obra era frecuente que las mujeres enseñaran, a cambio de una remuneración ínfima, labores de costura a las jóvenes para que éstas pudieran confeccionar sus propias prendas y venderlas. El problema de este trabajo artesanal era que algunas mujeres francesas compraban dichas prendas a precios muy bajos y las revendían más caras en sus tiendas, llevándose el máximo beneficio. Esta situación se daba principalmente en las zonas urbanas, pero en las rurales la situación no era mucho mejor. En zonas donde había ovejas, las madres enseñaban a sus hijas a trabajar en el telar con lana y seda, pero los tapices resultantes se vendían a un precio más bajo de lo que costaba fabricarlos. En estos casos, además, las mujeres solían ser las únicas trabajadoras del hogar, ya que los varones de sus familias preferían no hacerlo, de modo que dependían de ellas. Por ende, eran frecuentes los casos de abuso y explotación familiar.

En sociedades beduinas las mujeres participaban de las labores agrícolas, como los hombres, pero solían tener tareas más complicadas que ellos, como buscar agua en el desierto. Por este motivo, el autor critica que el trabajo de las mujeres no estuviera bien pagado, que se las relegara a puestos sin responsabilidad donde eran mano de obra esclavizada y sin descansos ni permisos por maternidad. Estos trabajos consumían mucho tiempo, pero el salario era insuficiente para mantener sus familias y el no tener acceso a una buena educación las condenaba a no poder optar a mejores puestos.

A lo largo de toda su obra, al-Ḥaddād se muestra como un férreo adversario del velo y el enclaustramiento, dedicando un gran apartado a combatir dichas costumbres, impuestas a las mujeres sin tener una base religiosa. En este sentido, en la obra se citan las numerosas y nefastas consecuencias que tienen estas costumbres para ellas y para la sociedad en general, puesto que, entre otras cosas, impiden que los esposos se conozcan, facilitan la suplantación de identidad, evitan que las mujeres puedan trabajar, las obligan a recurrir a un representante varón para que se encargue de sus bienes (de los que, según el Corán, la mujer debe tener disposición absoluta), etc. El velo es, además, causa de muchas de las desviaciones morales de tipo sexual que aquejan a la sociedad tunecina, puesto que al hallarse tan separados ambos sexos es imposible que se desarrolle una sexualidad natural y armoniosa. Asimismo, es improbable que una mujer enclaustrada y velada sepa educar a sus hijos en los valores de la nación y la libertad, puesto que ella misma los desconoce. 
Por estos motivos, para el autor es imperativo que se supriman radicalmente tanto el enclaustramiento como el velo. Al-Haddād combate la opinión, defendida por los detractores de esta liberación, de que el desvelo conlleva el libertinaje y la falta de moral de las mujeres, aduciendo a esta situación dos causas mucho más profundas y antiguas: la falta de una educación moral sólida y la decadencia moral de los hombres.

Țāhar al-Ḥaddād dedica una gran parte de su obra a mencionar todos aquellos aspectos relativos al matrimonio que considera injustos para las mujeres. Uno de los que denuncia con mayor fuerza es la costumbre de los padres de escoger esposos para sus hijas sin su consentimiento y sin darles la posibilidad de conocerlos antes de casarse con ellos y tomar la decisión definitiva. En primer lugar, esto contradice las leyes islámicas, como se vio en el apartado anterior, y en segundo lugar, genera una situación en muchas ocasiones muy denigrante para la mujer, obligada a mantener relaciones sexuales con un desconocido al que no ama, al que se halla sometida por el contrato matrimonial y cuya voluntad debe cumplir al mismo tiempo que lleva la casa, educa a los hijos, soporta a sus suegros y, en muchos casos, trabaja hasta la extenuación. La esposa no tiene derecho a negarse a los deseos del marido, de forma que no puede rechazar la intimidad sexual o su voluntad de contraer matrimonio con una segunda mujer. Tampoco puede criticarlo si éste le es infiel, pero, por el contrario, ella será duramente castigada ante el menor rumor que ponga en entredicho su fidelidad a su esposo o su honor. Esto es un grave crimen no sólo contra la mujer, sino también contra la ley divina, tal y como denuncia al-Haddād, puesto que impide a las mujeres ser sujetos independientes, las subyuga a la autoridad paterna o de sus maridos y las obliga a soportar abusos constantes.

Otro objeto de análisis son las costumbres sociales relativas al matrimonio, como las elevadas dotes que los padres exigen a los pretendientes de sus hijas, la presión social que sienten las familias por hacer una fiesta grandiosa, la necesidad de pagar los banquetes, etc. Según el autor, esta situación es insostenible ya que la mayoría de familias carecen de medios económicos suficientes para cubrir todos estos gastos, por lo que suponen un obstáculo para el matrimonio y llevan a muchas familias a la ruina. El matrimonio de las jóvenes impúberes es criticado en la obra, donde el autor recalca la necesidad de establecer una edad apropiada para que éste sea válido y de anular cualquier compromiso matrimonial en que una joven impúber esté involucrada. De modo semejante se critica el matrimonio de hombres que ocultan enfermedades transmisibles, por lo general venéreas, a la hora de firmar el contrato matrimonial. Este hecho, 
además, constituye un ocultamiento de verdad por lo que también incurre en un delito contra la ley islámica.

El último aspecto relativo al matrimonio que al-Haddād considera negativo para las mujeres es el de la sexualidad. En su opinión, hay muchos jóvenes recién casados que, convencidos de que sus esposas los aman, las fuerzan a mantener relaciones sexuales con ellos. Sin embargo, para las mujeres esto constituye, en realidad, un acto de violación entre cónyuges, ya que las jóvenes no conocen a sus maridos y, en muchos casos, no desean compartir el lecho con ellos. Los hombres, frustrados y hastiados del rechazo de sus esposas, recurren a nuevas mujeres normalmente procedentes de clases bajas, con las que se casan por un breve periodo de tiempo y luego las abandonan o rompen el matrimonio.

Esto ocurre especialmente entre hombres de clase burguesa y jóvenes de familias pobres, puesto que la mayoría de hijas de clase pudiente no son entregadas a hombres que ya están casados, pero las mujeres que proceden de capas sociales desfavorecidas ven en el matrimonio la única manera de mejorar en la escala socioeconómica. Estas jóvenes, en la mayoría de los casos, no tienen otra opción para salir de la miseria, por lo que acceden a matrimonios polígamos, frecuentemente engañadas. Sin embargo, una vez divorciadas, su situación empeora ya que nadie quiere casarse con ellas al haber perdido la virginidad.

Por último, al-Ḥaddād estudia ampliamente la cuestión de la educación, quejándose de lo básica que es y de las pocas opciones que tienen las mujeres de formarse en cualquier ámbito que no sean los trabajos tradicionales. Para él, la educación es la mayor necesidad humana en la vida. La grave ausencia de escuelas en árabe a las que puedan acudir mujeres preocupa al autor, ya que al verse obligadas a acudir a colegios franceses y cristianos las mujeres sufren un proceso de aculturación que les impide desarrollar una mentalidad crítica con el colonialismo y un sentimiento nacionalista que transmitan a sus hijos. Al-Ḥaddād se enfrentó a duras críticas por estos postulados, pero rechazó cualquier idea que supusiera un límite para la educación de las mujeres acusándola de retrógrada y culpándola de perpetuar el atraso de la población tunecina. Para él, la mujer necesitaba, siempre como madre de la nación y pilar de la sociedad, una educación consolidada que la dotara de las herramientas necesarias para desarrollar una personalidad madura y fuerte, con un buen conocimiento de sus derechos y deberes.

\section{CONCLUSIONES}

Como se ha venido sugiriendo, las causas del atraso de las mujeres tunecinas se hallan, principalmente, en el atraso de la sociedad tunecina, que establece unas 
reglas sociales muy estrictas para las mujeres y les impide alcanzar la libertad, y en una interpretación patriarcal del islam. Para el autor no se trata únicamente de una cuestión de género, sino también de una cuestión de poder mediante la cual los hombres, para hacer prevalecer su hegemonía, imponen a las mujeres unas costumbres claramente discriminatorias. Reformar la sociedad, introducir en ella valores positivos y cambios que permitan a las mujeres una mayor integración en la vida pública e intelectual y el poder de decidir libremente sobre sus propios asuntos, es una necesidad a la que el pueblo tunecino debe dar respuesta de inmediato si desea alcanzar el progreso y la verdadera modernidad. Por todo lo anterior, esta obra no sólo constituye un referente para la liberación de la mujer árabo-musulmana, sino también un documento que permite el estudio de la situación de la mujer tunecina en la época del autor y da una visión general de los temas que preocupaban al movimiento de liberación femenino y a la sociedad en general. El inmenso rechazo con que fue recibida da fe de la división existente en la sociedad entre partidarios de los derechos de la mujer y detractores tradicionalistas.

Al-Haddād critica severamente el conservadurismo religioso y acusa a sus seguidores de tenerse por defensores del islam cuando en realidad lo usan como excusa para impedir el avance de la sociedad. Ésta y otras cuestiones de índole ideológica llevaron a muchos ulemas y šuyūj de al-Zaytūna a criticar ampliamente la obra, incluso admitiendo no haberla leído nunca. A causa de las intensas críticas que el libro suscitó, al-Haddād fue amenazado en múltiples ocasiones por grupos de jóvenes radicales que incluso lo atacaron personalmente, por lo que pasó el resto de sus días recluido en casa no sólo debido a su enfermedad (padecía una enfermedad cardíaca y posteriormente contrajo tuberculosis), sino también a causa de dichas amenazas.

El libro fue publicado por primera vez en 1930, pero fue rápidamente vetado y retirado de venta y hubo que esperar 1978 para una nueva edición, cuando la situación estaba más calmada y el clima era propicio. Por todo lo anterior, podemos constatar la importancia de este autor en el incipiente feminismo tunecino y en el ámbito de la reforma social y su influencia en la posteridad, como demuestran el Código de Estatuto Personal de Túnez (para más información sobre este código y, más concretamente, sobre el estatus jurídico de las mujeres en Túnez, ver Chaouachi 191-2078, Pérez Beltrán 327-335 y Ruiz-Almodóvar 157-199), basado en algunas de sus ideas; la instauración, a partir del gobierno de Būrgība, de un régimen que podríamos considerar como feminismo institucional o la proliferación de movimientos y asociaciones culturales y de liberación de la mujer en Túnez. 
En cuanto al papel de la religión en el pensamiento de al-Ḥaddād, resulta evidentemente central. Como hemos visto, la religión es el hilo conductor que articula las propuestas del autor, que se mueve siempre dentro de los esquemas del reformismo islámico, siguiendo la estela de otros pensadores ya mencionados. Si bien sus ideas pueden resultar revolucionarias, frecuentemente aparecen respaldadas con aleyas coránicas, lo que deja claro que el autor se preocupa por cumplir sus obligaciones como creyente y por seguir la voluntad de Dios. El autor maneja el texto sagrado principalmente como fiel y después como reformista. Lo interpreta, usando su libertad de albedrío, de tal forma que se adapta a las necesidades del momento y, evidentemente, en respaldo de sus propias premisas.

Además, a lo largo de toda su obra podemos ver claramente los dos roles principales que al-Haddād concibe para las mujeres y que están muy vinculados con el ambiente reformista islámico del momento: por un lado, la mujer como madre, como pilar de la familia y como perpetuadora de la fe y la moral religiosa; por otro lado, la mujer como educadora de los hijos, como transmisora de conocimientos y creadora de consciencia. Si bien es cierto que ambos roles presentan claras rupturas con respecto a la visión tradicionalista de los mismos (la mujer como madre reducida a la labor procreadora, como objeto pasivo, frente a esta madre activa que al-Haddād propone; la mujer como depositaria de la moral y el honor frente a la mujer como luchadora por la unidad nacional y la libertad de Túnez a través de la creación de ciudadanos conscientes), se hallan estrechamente vinculados con el fondo religioso presente en toda la obra de al-Haddād. No podemos olvidar que, si bien fue un gran reformista y adalid de la liberación femenina en su país, este pensador recibió una educación eminentemente tradicionalista tanto en la escuela coránica como en la Gran Mezquita al-Zayt ūna, por lo que, aunque se empapara de ideas nuevas que llegaban de ámbitos seculares y occidentales, sus teorías siempre partían de ese marco islámico del que hablábamos al comienzo de este artículo.

\section{REFERENCIAS BIBLIOGRÁFICAS}

Borrmans, Maurice. «La Tunisie». Statut Personnel et famille au Maghreb de 1940 a nos jours. París y La Haya: Mouton, 1977, 120-153.

Chaouachi, Saida. «Le statut juridique de la femme en Tunisie». Droits de citoyenneté des femmes au Maghreb. Eds. Belarbi, Aicha, Fattouma Benabdenbi Djerrari, Saida Chaouachi et al.. Casablanca: Le Fennec, 1997, 191-207.

El Corán. Edición preparada por Julio Cortés. Barcelona: Herder, 2009.

Ḥaddād, Țāhar al-. Imrā̄tu-nā fi l-šañĩ a wa-l-muŷtamac. Túnez: Al-Dār al-Tūn̄̄siyya li-l-Našr, 1989. 
Haddād, Țāhar al-. Muslim Women in Law and Society. Annotated translation of al-Ṭāhir al-Haddād's Imra'tunā fi 'l-sharica wa l'-mujtamac', with an introduction. Trad. Ronak Husni y Daniel L. Newman. Londres y Nueva York: Routledge, 2010.

Pérez Beltrán, Carmelo. «Las leyes de familia de Túnez promulgadas durante la primera década del siglo XXI», Miscelánea de Estudios Árabes y Hebraicos 60 (2011): 327-335.

Ruiz-Almodóvar, Caridad. «El Código Tunecino de Estatuto Personal», Miscelánea de Estudios Árabes y Hebraicos 44 (1995): 157-199.

Sraieb, Noureddine. «Contribution à la connaissance de Tahar el Haddad (18991935)», Revue de l'Occident Musulman et de la Méditerranée 4 (1967): 99-132. 